\title{
A Simulation-based Approach for the Behavioural Analysis of Cancer Pathways ${ }^{\star}$
}

\author{
Agastya Silvina ${ }^{[0000-0002-0012-9256]}$, Guilherme Alfredo Redeker ${ }^{[0000-0002-8722-2559]}$, \\ Thais Webber ${ }^{[0000-0002-8091-6021]}$, and Juliana K. F. Bowles ${ }^{[0000-0002-5918-9114]}$ \\ School of Computer Science, University of St Andrews, St Andrews, KY16 9SX, UK \\ $\{$ as362, gr60, tcwds, jkfb\}@st-andrews.ac.uk
}

\begin{abstract}
Cancer pathway is the name given to a patient's journey from initial suspicion of cancer through to a confirmed diagnosis and, if applicable, the definition of a treatment plan. Typically, a cancer patient will undergo a series of procedures, which we designate as events, during their cancer care. The initial stage of the pathway, from suspected diagnosis to confirmed diagnosis and start of a treatment is called cancer waiting time (CWT). This paper focuses on the modelling and analysis of the CWT. Health boards are under pressure to ensure that the duration of CWT satisfies predefined targets. In this paper, we first create the visual representation of the pathway obtained from real patient data at a given health board, and then compare it with the standardised pathway considered by the board to find and flag a deviation in the execution of the cancer pathway. Next, we devise a discrete event simulation model for the cancer waiting time pathway. The input data is obtained from historical records of patients. The outcomes from this analysis highlight the pathway bottlenecks and transition times which may be used to reveal potential improvements for CWT in the future.
\end{abstract}

Keywords: Cancer pathway $\cdot$ Cancer waiting time $\cdot$ Discrete event simulation Process modelling

\section{Introduction}

Cancer is a condition where cells in some part of the human body reproduce at an uncontrollable rate. The cancer cells, instead of working together with the system, become another entity of the body, which could potentially jeopardise human health and wellbeing [6]. Patients with chronic cancer (e.g., lung cancer) have to undergo a series of treatments to attempt the eradication of the disease [10].

In the UK, before a patient undergoes a set of procedures that have been agreed upon by the board members and consultants, there is a period of waiting, usually ranging from 14 to 62 days $^{1}$. This period of waiting before the decision to treat the patient is known as Cancer Waiting Time (CWT) and has several planned activities depending on the patient's health condition, further tests evaluations and medical board meetings ${ }^{2}$.

\footnotetext{
* This research is partially supported by the DataLab.

${ }^{1}$ https://www.england.nhs.uk/wp-content/uploads/2015/03/delivering-cancer-wait-times.pdf

${ }^{2}$ Standards: https://www.isdscotland.org/Health-Topics/Waiting-Times/Cancer/Guidance/
} 
The CWT pathway for lung cancer treatment provided by the UK National Health Service (NHS) board is illustrated on Figure 1. Within the first time period (first 2 weeks), patients will be referred by their general practitioner (GP) and then undergo several tests. During the next 14 to 28 days, the patient will have further tests. Once the board agrees on the first treatment, the decision will be made within the next 28 to 62 days. Usually, the waiting time for a decision should not exceed a 62 days period. However, there are many cases when that happens. Further, lung cancer has a poor prognosis: over half of people diagnosed with lung cancer die within one year of diagnosis and around $17.8 \%$ within the 5-year survival [10]. Hence, it becomes necessary to decrease the waiting time from the GP referral to their first treatment.

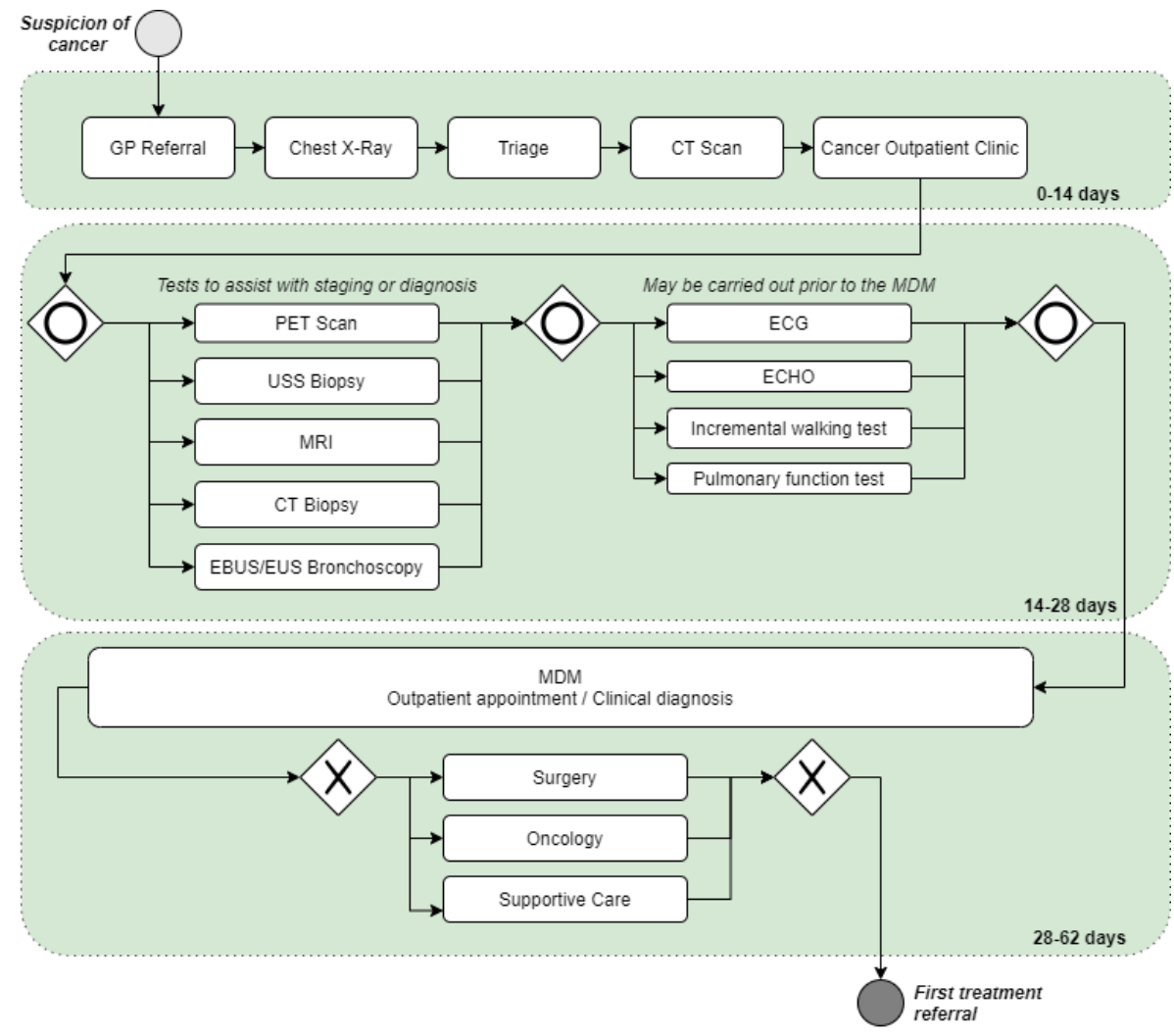

Fig. 1. Lung Cancer Pathway based on NHS Board

By streamlining the process of analysing the CWT dataset (e.g., through simulation) and comparing the 'actual' pathway patients experience versus what the NHS Board considers the pathway to be, we can highlight bottlenecks and transition times that exhibit high variability across patients. These insights will provide quantitative evidence that might be useful to design follow-up interventions. For example, policies to 
reduce the variability of specific transition times between activities may lead to overall improvements in CWT and reduction of the cumulative delays.

Discrete event simulation (DES) has been widely used in many sectors including for healthcare processes management [3], [5], [8]. DES allows healthcare professionals, for instance, to assess the efficiency of the existing healthcare delivery system. It has many usages ranging from forecasting the impact of changes in patient flow [5], examining the resources needed and available within a hospital [4], to observing the improvement of patient experience in an emergency department [1]. Related to this work, a DES model has been developed in the field of radiation therapy to reduce waiting time as well as to improve the treatment process planning [2]. Another recent work [7] focused on patient scheduling decisions related to chemotherapy in order to efficiently use medical resources and provide timely access to cancer treatment. Hence, DES provides the ability to investigate the complex relationship between various parameters (e.g., patient arrival rates and activities service times) through a stochastic model able to produce statistical estimates on the metrics of interest (e.g., waiting time).

The entities in a DES model (e.g., patients) are visiting service stations that compose the process, and sometimes they need to wait until service can be provided. This means that the demand for service has exceeded the capacity to provide the service readily. This information allows the healthcare professional to observe operational processes, simulating alternatives that can be used to reconfigure the existing pathway, to improve its performance, and to plan (change or reduce) its activities, without altering the present process execution while conducting such scenarios experiments [5], [8]. By treating lung cancer care from the first GP referral to the first treatment as a series of discrete events, we can simulate the cancer pathway with a DES approach allowing us to understand the overall treatment process, its complexities, patient journey and bottlenecks during the pathway.

This paper is structured as follows. In Section 2, we present the CWT dataset and the extraction of quantitative information through database queries development. The simulation modelling approach, the generated pathway and input modelling are discussed in Section 3. In Section 4 we present the approach used to validate our simulation model. Section 5 discusses the simulation scenarios and the findings. We conclude with suggestions for further work in Section 6.

\section{Data analysis and queries development}

To analyse the cancer pathway dataset compatibility with the steps established by the cancer pathway guideline as shown in Fig. 1, we acquired the dataset for lung cancer from three different hospitals within an NHS health board. The dataset contains 642 patients from 2016 to 2019 with lung cancer within NHS Lothian. It gathers the information regarding the outpatient (i.e., patient not formally admitted to the hospital), inpatient treatment (i.e., patient formally admitted to the hospital), and patients' orders (e.g., prescriptions, test's appointments). It also contains the information related to the type of the event (e.g., services for the outpatients, order_item for orders), the event start date (i.e., when the event is registered to the system), event execution date (i.e., the exact start date of the event) and event finish date. 
The modelling effort proposed in this paper aims to help the health professionals to analyse lung cancer pathway behaviour as a complex process (CWT) and assess its execution issues. For the first milestone, we focus on the whole observed waiting time without splitting the events based on each waiting time category (i.e., 0-14 days, 14-28 days, 28-62 days). Even though the board states the splitting, in reality, patients do not strictly follow the procedure guideline.

The guideline establishes that several tests could be performed prior to a final decision on the treatment, at any time, and it is up to the medical professionals to define the ones appropriate to each case following the patient's case development. The overall care process is rather complex and could take considerable time to schedule appointments and tests, reschedule, execute procedures and analyse/deliver results, depending on available resources and specific test characteristics. Thus, after discussions with healthcare professionals, we use the dataset to describe the behaviour of the CWT process, and then use the gathered information to create the DES model. We are interested in calculating the time patients spend on (parts of) the pathway (i.e., added time), and the number of patients flowing throughout the process (i.e., between activities), as well as calculate the elapsed days according to each milestone on the guideline, as follows:

- We calculate the number of patients in the dataset and the added service time observed between the first GP Referral to the definitive Multidisciplinary Meeting (MDM). MDM is an important event that happens at least once before starting the patient's treatment. In this meeting, the healthcare professionals determine whether a patient needs further care. The definitive MDM is the last MDM before starting the patient' treatment (e.g., radiotherapy, surgery, palliative care).

- We calculate the number of patients undergoing each category of tests requested (i.e., CT Scan, CT Biopsy, PET, Bronch/EBUS, Surgical staging/Biopsy).

- We calculate the added service time observed from the first outpatient (OP) appointment (in the Cancer Outpatient Clinic) to the definitive MDM.

- We calculate the number of patients who have more than one outpatient (OP) appointment prior to the definitive MDM.

- We calculate the added service time observed between Triage to the first OP appointment.

- We calculate the service time per order, i.e., for each test: CT Scan, PET Scan, CT Biopsy, grouped by the type of first treatment given to the patients.

We design the SQL queries to get the information directly from the Trak Oracle database to calculate each requested observation. The design of these queries is similar to each other. First, we categorise the event based on the service (i.e., for the outpatient) and order_item (i.e., for orders). There are more than 1,000 different services and order_items. However, there is no list to categorise these events. Together with the health professionals, we created a list of categories. For some services/order_items, we use keywords (e.g., CT, PET). Once we get the list of the services/order_items (event), we perform a manual check. For the other events without a reliable keyword, we choose the event that was performed to a significant number of patients, i.e., more than 50 patients.

- Once we categorise the service, we calculated the elapsed days between the categorised events and the first treatment date. 
- With the elapsed days, we determine the first (or last) events (i.e., the definitive MDM is the one which has the highest elapsed days).

- Hence, we can calculate the added service time and the number of patients between various events.

From these observations we can derive an observed pathway which is the basis of our simulation model.

\section{Simulation modelling and DES}

After analysing the dataset, we simulate the CWT process by creating a discrete event simulation (DES). We want to compare the pathway in the guideline (refer to Fig. 1) and the pathway that emerged from the data. By knowing the difference between the two, we can then further investigate and perform analysis for the CWT process within the health board. In this case study, we applied a simulation modelling process [8] in four basic steps (problem statement, process activities identification, flow analysis, metric(s) of interest, and model refinement) as follows.

1. Problem statement: we aim to simulate the pathway from the first time patients are being referred by their general practitioner (GP)/the first time the patients are seen in the hospital until the start of their first treatment. The time elapsed between these two events is defined as cancer waiting time. We are interested in finding the events/occasions when elapsed days exceed 62 days (i.e., the recommended maximum days for cancer waiting time according to the guideline).

2. Process activities and execution flow: we abstract the activities and process behaviour from the guideline as shown in Fig. 1 as well as matching with the available CWT data. We determine the composition of the process in terms of entities (e.g, patients) and activities/events (e.g., CT Scan, MDM, and so on) that we assign into the appropriate simulation flow. The process starts with patient arrival event (GP referral) and ends with first treatment referral, for each patient. Each activity in the process represents a service (an event) to a patient performed within a service time distribution. The process flow can contain one or more decision gateways, each indicating two or more output probabilities (or conditions) to follow. In our case, we present two gateways ruled by probabilities. The event scheduling process of DES tools[8] presents a time advance mechanism that guarantees that at least one event is scheduled when simulation starts and that it will consume an event list during execution. The simulation execution captures quantitative information until a defined stop criteria is reached. Usually, the arrival event configuration dictates the simulation continuance (or halt), i.e., the maximum number of entities arrivals to simulate. Also a desired simulation length (e.g., duration in days) can be defined prior to execution.

3. Metric(s) of interest: once all the activities of the process (i.e., events in the model) have been defined, we choose the performance metric to assess within our simulation (i.e., waiting time, elapsed days between activities, and so on). Variables and counters can be coded by the simulation analyst to provide additional quantitative results on the sample journeys, as long as required. 
4. Model refinement: after the process flow and activities have been defined, we indicate the model parameters. They represent fitted probability distributions to be applied at each activity (event) determining their behaviour expressed as service times, and as inter-arrival time regarding the patients arrival (GP referrals). The output probabilities on gateways are acquired from further statistical analysis over the dataset concerning all patients' journey. Once these timing information are set for each component in the DES, we can run the model and perform refinements on parameters, counters, variables, and overall behaviour to match the actual process according to the validation framework adopted, until we have a coherent base model checked through a statistic measurement of the dataset.

Before we simulate the CWT, we simplify the pathway as shown in Fig. 2. The focus is on finding the process bottleneck that may represent delays on the most common procedures (events) the patients undergo from their first GP appointment to their first treatment. Hence, for our model, we choose several events during the CWT pathway based on the number of occurrences and categorised them in four activities in which a patient can be at any time: (1) First appointment (First OP), such as Triage, Cancer Outpatient appointment; (2) CT Scan (i.e., primary test before further tests); (3) Most common further tests (i.e., CT Biopsy and PET Scan); and (4) MDM. The GP referral and First treatment referral are considered as start and end events in the pathway, respectively. After choosing and categorising these events, we update the current pathway. Fig. 2 shows the simplified pathway with activities and events within the milestones.

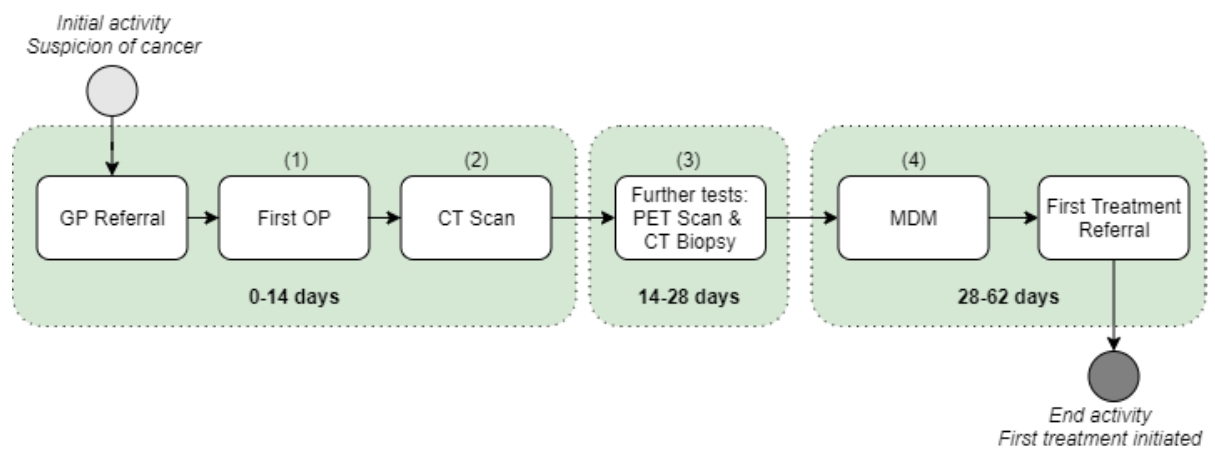

Fig. 2. Simplified lung cancer pathway obtained by dataset analysis

Further, we compare the simplified pathway with the pathway captured from the dataset. From the dataset, we find that the CWT does not strictly follow the guideline regarding the pathway. Instead, the pathway is an iterative process as shown in Fig. 3. From our observation, any event can happen in any particular order. Tests, appointments, and discussions are events that may happen several times in a patient journey.

Thus, Fig. 3 also represents the DES model. The start event ruled by patient interarrival time distribution is GP referral. The end event ruled by a probability within an exclusive decision gateway is First treatment referral. The remaining activities in the 


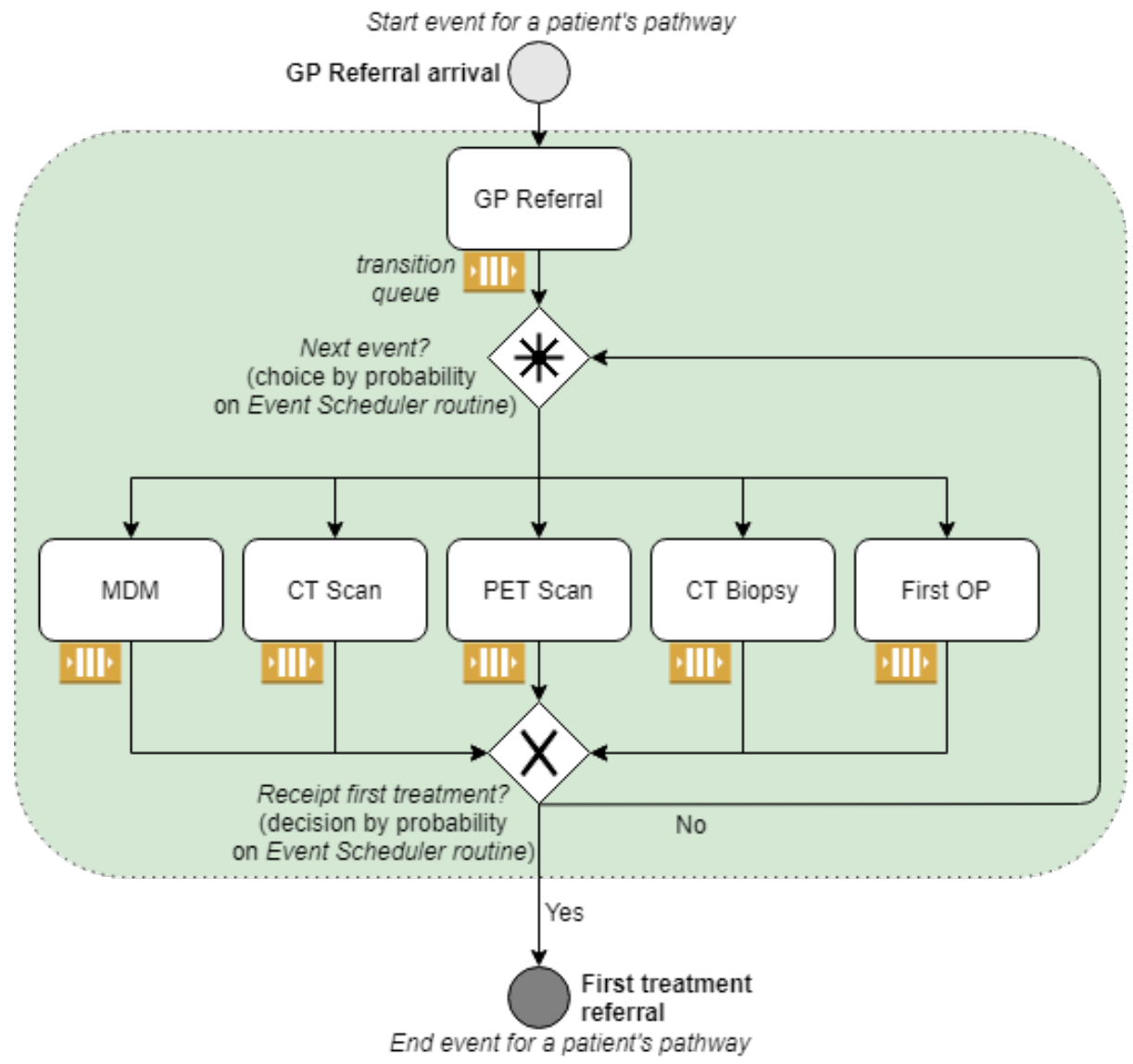

Fig. 3. Observed lung cancer pathway as a simulation (DES) model

model are First OP, MDM, CT Scan, CT Biopsy, PET Scan. These activities represent scheduled events in the simulation core, and at each iteration they are also computed as states reached during execution to further compile the entities routing statistics. We added queues in the simulation behaviour to reproduce the actual CWT accumulated delays, i.e., to buffer patients on each activity thus simulating the transitions where the patients do not undergo any tests, appointments, or MDM but surely they are buffered waiting for their next activity in the pathway.

We focus on determining the process activities (or in this case, also its events) and the process flow (i.e., including activities partial ordering) as the other simulation parameters and components are correctly handled by the simulation framework Salabim ${ }^{3}$.

Salabim is an open-source object-oriented developed for DES of complex control in logistics and production environments. It follows the methodology of process descrip-

\footnotetext{
${ }^{3}$ More information on simulation framework can be found on https://www.salabim.org/
} 
tion as demonstrated in Simula and later in Prosim, Must and Tomas. As a python package, it allows the use of other powerful python libraries (e.g., for statistical processing, presentation, machine learning). It also has an integrated animation engine which eases the manual observation of process flow for the user (i.e., the healthcare professionals).

The model description we presented in Fig. 3 is coded within the Salabim simulation core, which is illustrated in Fig. 4 with important routines and main program flow.

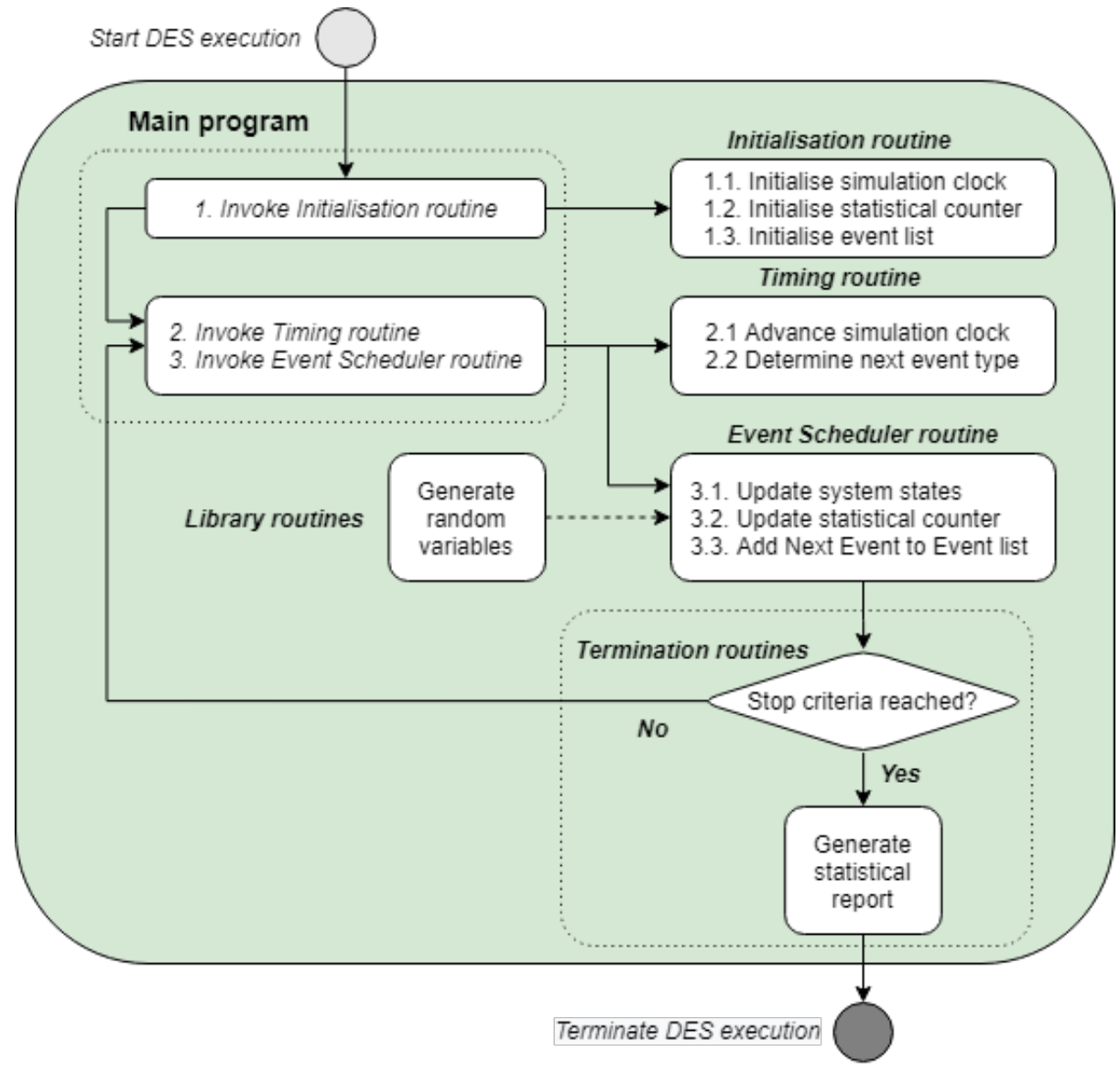

Fig. 4. Simulation core flow (DES routines)

To determine the distributions for the simulation parameters, we use the KolmogorovSmirnov test (kstest). The kstest compares the two sample statistical distributions. It is a non-parametric test which does not require the data to follow the normal distribution. When we compare the possible distributions (e.g., normal, exponential) to the dataset, we get the $D$ statistic and $p$ value. The $D$ statistic is the maximum absolute difference between two distribution functions. By comparing the $D$ statistic and $p$ value for each 


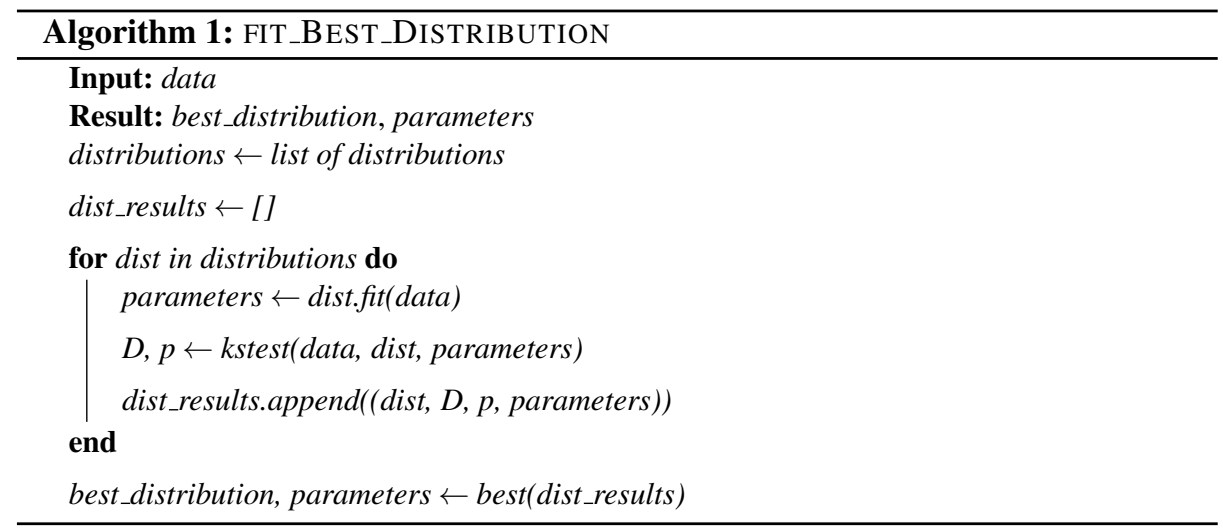

distribution (i.e., minimum value), we can determine the best fit distribution for the data. The procedure is shown in Algorithm 1. The list of distributions is composed of 12 distributions, i.e., we identified six types of events, and for each event, we fit two distinct distributions. One distribution for the time needed for patients to undergo the particular event, and the second distribution is for the transition time between the current event to the next one. Table 1 shows each activity and its service time distribution provided by the dataset statistical analysis.

Table 1: Cancer Waiting Time pathway simulation information

\begin{tabular}{|l|l|}
\hline \hline EVENTS/ACTIVITIES & FITTED PROBABILITY DISTRIBUTIONS \\
\hline \hline GP Referral arrival & Interval time: norm $(2.33,1.76)$ \\
\hline GP Referral transition & Interval time: genextreme $(-0.36,4.77,4.86)$ \\
\hline First OP & Service time: constant $(1$ day $)$. \\
\hline First OP transition & Service time: genextreme $(-0.23,8.53,7.56)$ \\
\hline CT Scan & Service time: genextreme $(-0.73,4.70,4.73)$ \\
\hline CT Scan transition & Service time: genextreme $(-0.45,7.25,6.27)$ \\
\hline CT Biopsy & Service time: exponweib $(1.47,1.94,-0.997,14.92)$ \\
\hline CT Biopsy transition & Service time: genextreme $(-0.13,6.36,4.18)$ \\
\hline PET Scan & Service time: genextreme $(-0.037,13.69,4.29)$ \\
\hline PET Scan transition & Service time: genextreme $(-0.13,6.36,4.18)$ \\
\hline MDM & Service time: constant $(1$ day $)$ \\
\hline MDM transition & Service time: genextreme $(-0.42,8.65,9.18)$ \\
\hline \hline
\end{tabular}

The execution flow (refer to Fig. 3) schedules events to patients in a FIFO (Firstin/First-out) policy, and the flow can reach two possible decision gateways. The first gateway represents a decision on the test(s) a patient will undergo during the pathway (i.e., schedule new event). This one determines, from the set of events, which one is the next to be scheduled in the simulation core, following the current simulation state (i.e., current activity the patient is undergoing) and simulation time counters. The second gateway determines a two-way probability that defines whether the entity (patient) will remain in the pathway executing activities (i.e., schedule new event), or leave the simulation since it received first treatment referral after MDM (i.e., schedule the end event). For the output probabilities, we calculate the number of transition from each activity (i.e., state). For each state, we set a different probability for determining the next state (e.g., according to the dataset, more than $50 \%$ of the patients will undergo First $O P$ after $G P$ Referral). 
Figure 5 show the transition probabilities matrix used within the first decision gateway. The second gateway presents a conditional probability determining if patient had at least one MDM in his/her pathway. According to dataset, $49 \%$ of patients get their first treatment after $M D M$.

\begin{tabular}{|c|c|c|c|c|c|c|c|}
\hline & GP Ref & First OP & CT Scan & PET Scan & CT Biopsy & MDM & First treatment \\
\hline GP Referral & - & $46 \%$ & $9 \%$ & $16 \%$ & $7 \%$ & $22 \%$ & - \\
\hline First OP & - & $23 \%$ & $36 \%$ & $7 \%$ & $4 \%$ & $9 \%$ & $21 \%$ \\
\hline CT Scan & - & $21 \%$ & $15 \%$ & $11 \%$ & $2 \%$ & $2 \%$ & $49 \%$ \\
\hline PET Scan & - & $23 \%$ & $19 \%$ & $6 \%$ & $4 \%$ & $8 \%$ & $40 \%$ \\
\hline CT Biopsy & - & $18 \%$ & $67 \%$ & $4 \%$ & $3 \%$ & $3 \%$ & $5 \%$ \\
\hline MDM & - & $18 \%$ & $72 \%$ & $2 \%$ & $5 \%$ & - & $2 \%$ \\
\hline First treatment & - & - & - & - & - & - & $100 \%$ \\
\hline
\end{tabular}

Fig. 5. Transition matrix with routing probabilities (\%)

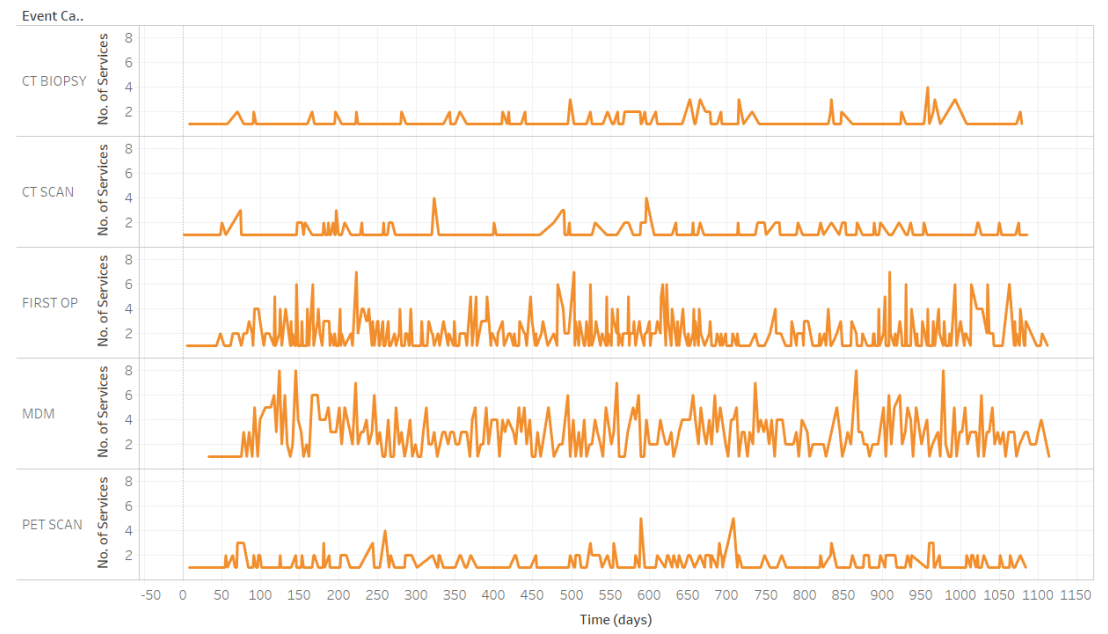

Fig. 6. Number of services for the CWT events

Even though our dataset contains no information regarding the number of resources (i.e., staff availability and the number of machines), we still incorporated the resources as parameters to guide the events scheduling. This way, we simulate varied scenarios of having more healthcare professionals and machines to the overall pathway activities. We calculate the number of resources by observing the maximum number of services and test orders that can happen in any days from our dataset. Fig. 6 shows the number of services for the event we selected from 2016 to 2019, i.e., 1,150 days (3-year dataset). Though these numbers may not be an accurate representation of the number of 
resources, we can use it as starting point before we obtain the information (e.g., from interviews, survey or new dataset) regarding the resources capacity and availability.

\section{Model validation}

The input data modelling provided fitted distributions to service times in all activities as well as patient inter-arrival rate concerning GP referrals. Also, the decision gateways probabilities are set with the compilation of all occurrences in the complete pathway, for all patients in the dataset. These input parameters were also discussed with healthcare professionals that actively participate in the CWT. After running the model for 365 simulated days, we observe the output (i.e., the sampling on the waiting time metric) in order to compare with real data, i.e., the average waiting time.

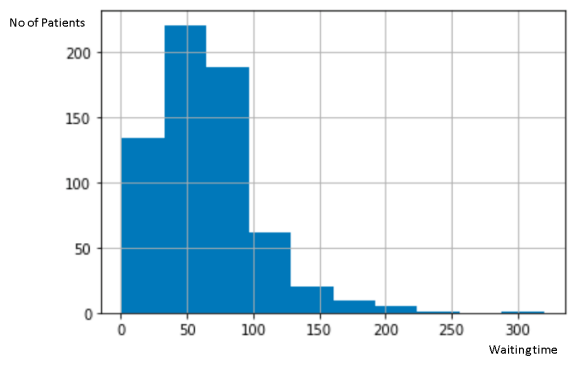

(a) Real patients' waiting time distribution

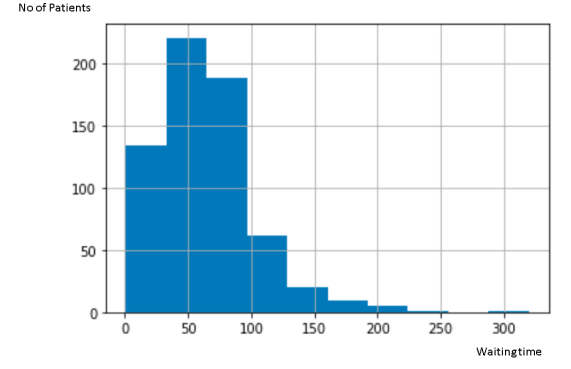

(b) Simulated patients' waiting time distribution

Fig. 7. Patients' waiting time distribution in days

Fig. 7 shows the waiting time distribution for both real data and simulation. Because the waiting time is not normally distributed, we use KS-test to compare both dataset distributions [9]. The result is $K S$-statistic $=0.06984$ and $p$-value $=0.152$. We know that if the KS statistic is small or the p-value is high, then we cannot reject the hypothesis that the distributions of the two samples are the same.

Table 2: Patients' waiting time descriptive statistic

\begin{tabular}{|c|c|c|}
\hline \hline MEASURES & ACTUAL (DATASET) & SIMULATED \\
\hline \hline Mean & 64.42 & 65.87 \\
\hline Std deviation & 37.67 & 39.04 \\
\hline 25\% percentile & 38 & 37 \\
\hline 50\% percentile & 61 & 56 \\
\hline 75\% percentile & 82 & 84 \\
\hline \hline
\end{tabular}

From the KS-test result, the $p$-value is high (more than 5\% level of significance). Hence, we cannot reject that the distributions of the two samples are the same. The result 
of the descriptive statistic for both datasets as shown in Table 2 supports the close correspondence between the simulated and observed outcome. Therefore, our model can provide reasonable approximation and observation of the expected system behaviour.

\section{Simulation Results}

The purpose of creating a DES model of the CWT pathway is to simulate alternative scenarios to analyse bottlenecks and assess how to improve the current process. Table 3 shows the simulation scenarios and their respective results. We built multiple simulation models varying parameters such as Service Capacity (SC), Waiting Transition Time (WTT), and Service Time (ST). Thus, for each scenario, we modify the number of resources available (e.g., the number of machines), the transition time needed between each event, and the time needed for the service execution, respectively. The applied total simulation length is 365 days to collect statistics.

Results demonstrate that increasing in 50\% the SC for MDM (Scenario (3)) or limiting the WTT after MDM in 5 days (Scenario (4)) we achieve the best improvements for patients, i.e., getting the first treatment before 62 days. Regarding Scenario (3), results show $62 \%$ of the patients started the treatment before 62 days whilst in the Scenario 4 the result is even better with $74 \%$ of patients simulated. These results show that MDM can be a bottleneck in the whole process because almost all patients will undergo MDM before getting their first treatment.

Table 3: CWT pathway simulated scenarios and their results

\begin{tabular}{|c|l|c|c|}
\hline \hline$\#$ & $\begin{array}{l}\text { Simulated SCENARIOS } \\
\text { (varying parameters SC, WTT, and ST) }\end{array}$ & $\begin{array}{c}\text { MEAN WAITING TIME } \\
95 \% \text { CI (lower, upper) }\end{array}$ & $\begin{array}{c}\% \\
(<62 \text { days })\end{array}$ \\
\hline \hline$(1)$ & $50 \%$ increase SC for First OP & $67.06(59.78,74.32)$ & 56 \\
\hline$(2)$ & limit WTT after First OP (5 days) & $63.99(56.72,71.25)$ & 60 \\
\hline$(3)$ & $50 \%$ increase SC for MDM & $59.85(53.89,65.82)$ & 62 \\
\hline$(4)$ & limit WTT after MDM (5 days) & $49.45(44.14,54.76)$ & 74 \\
\hline$(5)$ & $50 \%$ increase SC for CT Scan & $63.91(56.97,70.85)$ & 59 \\
\hline$(6)$ & decrease ST on CT Scan (max 5 days) & $64.44(55.75,73.13)$ & 59 \\
\hline$(7)$ & limit WTT after CT Scan (5 days) & $64.30(57.61,70.98)$ & 58 \\
\hline$(8)$ & $50 \%$ increase SC for CT Biopsy & $65.97(59.12,72.81)$ & 53 \\
\hline$(9)$ & decrease ST on CT Biopsy (max 5 days) & $69.56(58.72,80.40)$ & 51 \\
\hline$(10)$ & limit WTT after CT Biopsy (5 days) & $65.81(58.11,73.51)$ & 54 \\
\hline$(11)$ & $50 \%$ increase SC for PET Scan & $65.80(54.37,77.38)$ & 53 \\
\hline
\end{tabular}




\begin{tabular}{|l|l|l|c|}
\hline$(12)$ & decrease ST on PET Scan (max 5 days) & $65.03(53.03,77.05)$ & 53 \\
\hline$(13)$ & limit WTT after PET Scan (5 days) & $65.80(54.37,77.28)$ & 53 \\
\hline
\end{tabular}

We also observe that most delays are caused by the transition time instead of the service time/availability of resources (i.e., from the First $O P$ and $M D M$ ). This is possible to check on Table 1, for instance, regarding CT Scan activity with probability distribution genextreme $(-0.73,4.70,4.73)$ compared to CT Scan transition with distribution genextreme $(-0.45,7.25,6.27)$, where the service time may not be an issue in the pathway, but the transition delay between services. The later can include another tasks that may delay the CWT, like bureaucracy and scheduling, just to name a few.

Adding more resources (e.g., by increasing the service capacity) for CT Scan, PET Scan or CT Biopsy has less impact on the overall patient waiting time, according to the results from Scenario (5) to Scenario (13). However, comparing the improvements in those three tests services show that CT Scan has better results when adding more resources. Thus, maybe CT Scan has a bottleneck and it is the test activity that needs more resources to not exceed 62 days period. As shown in Table 3, the probability of $M D M$ or First $O P$ as the next state is higher when compared to the likelihood for the tests (i.e., CT Scan, PET Scan, CT Biopsy). Hence, the less impact.

Even though we can simulate the lung CWT events, our model has many limitations. First, we do not consider the other services (e.g., MRI, Head CT, Pulmonary function tests) by merging all of the other services into queue transition after the states. During the simulation run, due to the fitted distribution function (e.g., exponential, gen-extreme) the simulation result may contain more outliers compared to the dataset as shown in Fig. 7. Also, the number of resources in our model is based on estimations.

In the future, we can improve the simulation by incorporating more services and update the resource number and service time with the information given by the hospital (e.g., by interview or another dataset extraction). We can also improve the simulation by adding more detailed characteristics, such as adding the type of the first treatment of the patients. This may increase the simulation accuracy because the average waiting time is very based on the patients' first treatment, as shown in Table 3.

\section{Conclusion}

Overall, simulations scenarios can provide valuable information for improvements in the whole CWT process. Even with the model limitation such as the number of resources (e.g., number of CT Scan machines), we can highlight the process bottleneck related to the board meeting resources (e.g. doctors, professionals) availability and give a close observation regarding alternative scenarios for the lung CWT.

The main bottlenecks of the lung CWT are related to the board meeting, i.e., the MDM state and the MDM transition. Additionally, regarding the tests' activities we exposed that the CT Scan is the one which could improve the CWT if more resources were available. Once we can add more activities, such as other tests, in the pathway from collecting integral CWT dataset within hospital database, we can fine-tune the service 
time and number of resources. DES can help to provide insights on process bottlenecks and improve the overall cancer care for the lung CWT. In future, more detail should be added in the simulation model to gain a better understanding of the process behaviour and clarify the transition delays in CWT.

\section{References}

1. Abo-Hamad, W., Arisha, A.: Simulation-based framework to improve patient experience in an emergency department. European Journal of Operational Research 224, 154-166 (2013)

2. Aivas, I., Tomasz, B., Cao, J., D’Souza, D., Mahjoub, R., Rodrigues, G., Zaric, G., Lock, M.: Reducing patient wait times for radiation therapy and improving treatment planning: A discrete-event simulation model. Clinical Oncology 29, - (2017)

3. Bangsow, S.: Use cases of discrete event simulation - Appliance and research. Springer, Berlin, Heidelberg (2012)

4. Baril, C., Gascon, V., Miller, J., Bounhol, C.: The importance of considering resource's tasks when modeling healthcare services with discrete-event simulation: an approach using work sampling method. Journal of Simulation 11, 103-114 (2017)

5. Günal, M., Pidd, M.: Discrete event simulation for performance modelling in health care: a review of the literature. Journal of Simulation 4, 42-51 (2010)

6. Hochhauser, J.S.T.D.: Cancer and its management. Wiley-Blackwell, 6 edn. (2010)

7. Liu, E., Ma, X., Sauré, A., Weber, L., Puterman, M.L., Tyldesley, S.: Improving access to chemotherapy through enhanced capacity planning and patient scheduling. IISE Transactions on Healthcare Systems Engineering 9(1), 1-13 (2019)

8. Rossetti, M.D.: Simulation Modeling and Arena. Wiley Press, 2 edn. (2010)

9. Young, I.T.: Proof without prejudice: use of the kolmogorov-smirnov test for the analysis of histograms from flow systems and other sources. Journal of Histochemistry and Cytochemistry 25, 935-941 (1977)

10. Zappa, C., Mousa, S.A.: Non-small cell lung cancer: current treatment and future advances. Translational Lung Cancer Research 5(3) (2016) 